\title{
ON PRIMARY GROUPS WITH COUNTABLE BASIC SUBGROUPS
}

\author{
BY \\ PAUL HILL AND CHARLES MEGIBBEN(1)
}

1. Introduction. This paper contains proofs of the results announced in [5] concerning nonisomorphic pure subgroups of primary groups with prescribed socles. In fact, in the present paper, we strengthen and generalize the main theorem of [5]. In addition, we establish the existence of a surprising abundance of essentially indecomposable groups and give a rather simple and general construction of infinite reduced primary groups without proper isomorphic subgroups. Some consideration is also given to those primary groups having the property that all their subsocles support pure subgroups. Although our most important results relate to primary groups with countable basic subgroups, they can clearly be generalized to groups having $\aleph_{0}$ as their critical number. A less trivial generalization to primary groups having greater cardinality than their basic subgroups is mentioned in our final section. All in all, this paper might be considered as one further contribution toward demonstrating the complexity of the structure of uncountable primary groups; for example, our Theorem 2.7 constitutes the most striking evidence known to the authors of the inadequacy of the Ulm factors of an uncountable primary group in determining the group.

All groups are assumed to be primary abelian groups for some fixed prime $p$, and all topological references are to the $p$-adic topology. We follow the notation and terminology of [3]. The cardinal $2^{\text {ko }}$ is denoted by $c$. We shall call $S$ a subsocle of the primary group $G$ if $S$ is a subgroup of $G[p]$. A subsocle $S$ supports a subgroup $H$ if $H[p]=S$. A subsocle $S$ of $G$ will be said to be a discrete subsocle if $S \cap p^{n} G=0$ for some positive integer $n$ and a dense subsocle if $S$ is dense in $G[p]$ (relative to the $p$-adic topology of $G$ ). A noteworthy property of dense subsocles is the following: if $S$ is a dense subsocle of $G$ and $H$ is a neat subgroup of $G$ supported by $S$, then $H$ is a pure subgroup of $G$ (see [6]). Finally, recall that a group is said to be essentially indecomposable if every direct decomposition of the group involves a bounded summand.

2. Pure subgroups with prescribed socles. If $G$ is a countable reduced primary group and if $G^{1}=0$, then each subsocle of $G$ supports, up to isomorphism, a

Received by the editors April 27, 1965.

(1) This paper was written while the second author was an ONR Postdoctoral Research Associate supported by Office of Naval Research Contract Nonr-4683(00), NR 043-321. 
unique pure subgroup of $G$. In this section, we show that this is not at all the case if we relax the condition to that of $G$ having a countable basic subgroup. We begin with a technical lemma which will be used in this, as well as a later, section.

LEMMA 2.1. Let $S$ be a subsocle of a primary group $G$ and suppose, for an infinite cardinal $m$, that $\left[H_{\alpha}\right]_{\alpha \in A}$ is a collection of subgroups of $G$ such that

(i) $|A| \leqq m$;

(ii) $\left|p H_{\alpha} \cap S\right|=m$ for each $\alpha \in A$;

(iii) $\left\{H_{\alpha}[p], S\right\} \neq G[p]$ for each $\alpha \in A$.

Then there are $2^{|A|}$ linearly independent subsets $L=\left[g_{\alpha}\right]_{\alpha \in A}$ of $G$ such that, for each $\alpha \in A$,

(a) $O\left(g_{\alpha}\right)=p^{2}$;

(b) $g_{\alpha} \in\left\{H_{\alpha}, G[p]\right\}$;

(c) $p g_{\alpha} \in S$;

(d) $g_{\alpha} \notin\left\{H_{\alpha}, S\right\}$;

and such that no two distinct such subsets generate with $G[p]$ the same subgroup of $G$.

Proof. We may assume that $A$ is the initial segment of all ordinals less than $\mu$, where $\mu$ does not exceed the first ordinal of cardinality $m$. Now suppose that $\beta<\mu$ and that we have chosen $x_{\alpha}$ and $x_{\alpha}^{\prime}$ in $G$ for each $\alpha<\beta$ such that the set $\left[x_{\alpha}, x_{\alpha}^{\prime}\right]_{\alpha<\beta}$ is linearly independent and such that $g_{\alpha}=x_{\alpha}$ or $x_{\alpha}^{\prime}$ satisfies conditions (a)-(d) for each $\alpha<\beta$. Since $|\beta|<m,\left|\Sigma_{\alpha<\beta}\left(\left\{x_{\alpha}\right\}+\left\{x_{\alpha}^{\prime}\right\}\right)\right|<m$, and therefore, because of (ii), there is a subgroup $\left\{y_{\beta}\right\}+\left\{y_{\beta}^{\prime}\right\}$ of order $p^{2}$ in $p H_{\beta} \cap S$ having trivial intersection with the subgroup $\Sigma_{\alpha<\beta}\left(\left\{x_{\alpha}\right\}+\left\{x_{\alpha}^{\prime}\right\}\right)$. Choose $z_{\beta}$ and $z_{\beta}^{\prime}$ in $H_{\beta}$ such that $p z_{\beta}=y_{\beta}$ and $p z_{\beta}^{\prime}=y_{\beta}^{\prime}$. Since (iii) holds, there is a $w_{\beta}$ in $G[p]$ but not in $\left\{H_{\beta}[p], S\right\}$. Set $x_{\beta}=z_{\beta}+w_{\beta}$ and $x_{\beta}^{\prime}=z_{\beta}^{\prime}+w_{\beta}$. The set $\left[x_{\alpha}, x_{\alpha}^{\prime}\right]_{\alpha \leqq \beta}$ is linearly independent and $g_{\alpha}=x_{\alpha}$ or $x_{\alpha}^{\prime}$ satisfies conditions (a)-(d) for $\alpha \leqq \beta$. Hence the set $L=\left[g_{\alpha}\right]_{\alpha \in A}$ is a linearly independent subset of $G$ satisfying the conditions (a)-(d) for each $\alpha \in A$. Since the set $\left[x_{\alpha}, x_{\alpha}^{\prime}\right]_{\alpha \in A}$ is linearly independent, the subgroup of $G$ generated by $L$ and $G[p]$ definitely depends on the choice, for each $\alpha$, of $g_{\alpha}=x_{\alpha}$ or $x_{\alpha}^{\prime}$. The proof is now complete.

REMARK 2.2. If, in Lemma $2.1, K$ is a subgroup of $G$ such that $|K|<m$, then clearly $y_{\alpha}$ and $y_{\alpha}^{\prime}$ can be chosen such that $K \cap \Sigma_{\alpha \in A}\left(\left\{y_{\alpha}\right\}+\left\{y_{\alpha}^{\prime}\right\}\right)=0$. Hence we may assume that $K \cap\{L\}=0$.

THEOREM 2.3. Let $G$ be a reduced primary group having a countable basic subgroup. If $S$ is a proper dense subsocle of $G$ such that $|S|=c$, then $S$ supports $2^{c}$ isomorphically distinct pure subgroups of $G$.

Proof. Let $\left[H_{\alpha}\right]_{\alpha \in A}$ be a collection of not more than $c$ pure subgroups of $G$ supported by $S$. Since $|S|=c$ and since $G$ has a countable basic subgroup, $|p H \cap S|=c$ for any pure subgroup $H$ of $G$ supported by $S$. Thus conditions (i)-(iii) of Lemma 2.1 are satisfied with $m=c$, so there are $2^{|A|}$ linearly independent 
subsets $L=\left[g_{\alpha}\right]_{\alpha \in A}$ of $G$ satisfying the conditions (a)-(d) and such that no two distinct such subsets generate with $G[p]$ the same subgroup of $G$. Since $\{L\}[p] \subseteq S$, there is a pure subgroup $H_{L}$ of $G$ supported by $S$ and containing $L$. That distinct $L$ 's cannot belong to a common pure subgroup $H_{L}$ follows immediately from the fact that no two $L$ 's generate with $G[p]$ the same subgroup of $G$. Hence $S$ supports (at least) $2^{|\Lambda|}$ pure subgroups of $G$; and, therefore, $S$ supports $2^{c}$ pure subgroups of $G$.

To complete the proof of the theorem, one need only observe that

$$
|\operatorname{Hom}(H, G)|=c
$$

whenever $H$ is a pure subgroup of $G$ supported by $S$. It is enough to note that $\operatorname{Hom}(H, G)$ can be imbedded in

$$
\operatorname{Hom}(B, G) \cong \sum_{n=1}^{\infty} \sum_{q(n)}^{*} G\left[p^{n}\right],
$$

where $B$ is a basic subgroup of $G$ and $q(n)$ is a countable cardinal for each positive integer $n$.

REMARK 2.4. By Remark 2.2, it is evident that, in Theorem 2.3, there exist $2^{c}$ isomorphically distinct pure subgroups of $G$ supported by $S$ all containing a common basic subgroup of $G$. In the sequel, we leave it to the reader to supply similar remarks where appropriate (e.g. Theorems 4.1 and 6.3 below).

COROLARY 2.5. Suppose that $G$ is reduced and has a countable basic subgroup. If $S$ is a proper dense subsocle of $G$ having cardinality $c$, then $S$ supports a pure subgroup of $G$ which is not an endomorphic image of $G$.

It was shown in [4] that high subgroups of a primary group need not be isomorphic; yet the question as to whether there was some reasonable uniqueness theorem remained (see [8]). In that connection, we have

COROLlaRY 2.6. Suppose that $G$ is a reduced primary group with a countable basic subgroup such that $G^{1} \neq 0$. If $G$ is not a $\sum$-group, then in order for $G$ to have fewer than $2^{c}$ isomorphically distinct high subgroups, the continuum hypothesis must be denied.

It is well known that the Ulm sequence (see [3]) of a countable reduced primary group completely determines the structure of the group. It is, of course, also known that the corresponding result does not hold for uncountable groups. Our final theorem of this section demonstrates quite strikingly the deficiency of the Ulm sequence of an uncountable primary group.

THEOREM 2.7. Let $\Omega$ be a limit ordinal of cardinality not exceeding $c$ and suppose that $G_{\alpha}(0 \leqq \alpha<\Omega)$ is a sequence of unbounded primary groups without elements of infinite height each having a countable basic subgroup. Then there 
exist $2^{c}$ isomorphically distinct reduced primary groups each having $G_{\alpha}$ $(0 \leqq \alpha<\Omega)$ as its Ulm sequence.

Proof. Let $G_{\Omega}$ be a nonzero cyclic $p$-group. Then, by Theorem 38.2 in [3], there is a reduced primary group $G$ of length $\Omega+1$ and cardinality $c$ having $G_{\alpha}(0 \leqq \alpha \leqq \Omega)$ as its Ulm sequence. We can write $G[p]=S+G^{\Omega}[p]$, where $S$ is a proper dense subsocle of $G$ having cardinality $c$. Since $G$ necessarily has a countable basic subgroup, $S$ supports $2^{c}$ isomorphically distinct pure subgroups of $G$. Moreover, each of these pure subgroups is necessarily maximal in $G$ with respect to intersecting $G^{\Omega}$ trivially. Let $H$ be any such pure subgroup of $G$. It is clear that $H$ has length $\Omega$, and it is not difficult to prove that $H^{\alpha}$ is pure in $G^{\alpha}$ and that $G^{\alpha} / H^{\alpha}$ is divisible for each $\alpha<\Omega$. It can then be shown easily that $\left\{H^{\alpha}, G^{\alpha+1}\right\}$ is pure in $G^{\alpha}$ for each $\alpha<\Omega$. Since $\left\{H^{\alpha}, G^{\alpha+1}\right\}[p]=G^{\alpha}[p]$ for $\alpha<\Omega$, it follows that $G^{\alpha}=\left\{H^{\alpha}, G^{\alpha+1}\right\}$ for each $\alpha<\Omega$. Therefore, for $\alpha<\Omega$, we have

$$
H_{\alpha}=H^{\alpha} / H^{\alpha+1}=H^{\alpha} /\left(H^{\alpha} \cap G^{\alpha+1}\right) \cong G^{\alpha} / G^{\alpha+1}=G_{x},
$$

as desired.

3. Distinguished subsocles. Throughout this section, $G$ will denote a primary group without elements of infinite height and $\bar{B}$ will denote the torsion completion of $G$, that is, $\bar{B}$ is a closed $p$-group containing $G$ as a pure subgroup with $\bar{B} / G$ divisible.

Definition 3.1. A subsocle $S$ of $G$ is said to be distinguished if $\{G[p], \bar{S}\}=\bar{B}[p]$, where $\bar{S}$ is the closure of $S$ in $\bar{B}$.

A discrete subscole is distinguished if and only if $G=\bar{B}$. The next theorem gives a useful characterization in the case of nondiscrete subsocles and also provides us with an alternative intrinsic definition of distinguished subsocles as opposed to the extrinsic one just given.

THEOREM 3.2. A nondiscrete subsocle $S$ of $G$ is distinguished if and only if the closure of $H$ in $G$ is pure whenever $H$ is a pure subgroup of $G$ such that $H[p]$ is a dense subsocle of $S$.

Proof. Assume that $S$ is a distinguished subsocle of $G$ and let $H$ be a pure subgroup of $G$ such that $H[p]$ is dense in $S$. There will then be a direct summand $K$ of $\bar{B}$ which contains $H$ as a dense subgroup and has $\bar{S}$ as its socle. The closure of $H$ in $G$ is then just $G \cap K$. Since $S$ is distinguished, $\{G, K\}[p]=\{G[p], K[p]\}$ and this equality implies that $G \cap K$ is neat in $K$. But $(G \cap K)[p]$ is dense in $K[p]$, and therefore $G \cap K$ is pure in $K$. Thus, the closure $G \cap K$ of $H$ in $G$ is a pure subgroup of $G$.

Conversely, suppose the closure of each pure subgroup of $G$ supported by a dense subsocle of $S$ is again a pure subgroup of $G$. Let $H$ be a pure subgroup of $G$ such that $H[p]$ is dense in $S$. Choose $A$ to be a proper basic subgroup of $H$ 
and let $y \in H$ be an element of order $p^{2}$ not in $A$. Suppose that $x \in \bar{B}[p]$. We can find a pure subgroup $A_{1}$ of $G$ such that $A_{1}[p]=A[p]$ and such that $x+y$ is contained in the closure $\tilde{A}_{1}$ of $A_{1}$ in $\bar{B}$. Then $x \in\left\{G, \tilde{A}_{1}\right\}$. Since $G \cap \bar{A}_{1}$ is the closure of $A_{1}$ in $G$ and is therefore pure in $G,\left\{G, \bar{A}_{1}\right\}[p]=\left\{G[p], \bar{A}_{1}[p]\right\}$. Hence $x \in\left\{G[p], \bar{A}_{1}[p]\right\}=\{G[p], \bar{S}\}$, and we conclude that $S$ is distinguished.

In [7] a quasi-closed primary group was defined to be a reduced primary group in which the closure of each pure subgroup is again pure. Quasi-closed groups are necessarily without elements of infinite height, and we have as an immediate corollary of the foregoing theorem.

COROLlary 3.3. $G$ is quasi-closed if and only if each nondiscrete subsocle of $G$ is distinguished.

From Theorem 3.2 it follows that every distinguished subsocle must support a pure subgroup. Indeed an even stronger result is implied.

COROLLARY 3.4. If $H$ is a pure subgroup of $G$ and if $S$ is a distinguished subsocle of $G$ such that $H[p] \subseteq S$, then $S$ supports a pure subgroup of $G$ which contains $H$.

Proof. We can construct a direct sum $A$ of cyclic groups in $G$ such that $A \cap H=0, A+H$ is pure in $G$ and $(A+H)[p]$ is a dense subsocle of $S$. The closure $M$ in $G$ of $A+H$ is pure since $S$ is distinguished. We need only choose $K$ neat in $M$ such that $A+H \subseteq K$ and $K[p]=S$.

As we have just shown, every distinguished subsocle supports a pure subgroup Among those subsocles of $G$ that do support pure subgroups, the next theorem characterizes those that are distinguished.

THEOREM 3.5. Suppose that the subsocle $S$ of $G$ supports a pure subgroup $H$ of $G$. Then $S$ is a distinguished subsocle of $G$ if and only if $G / H$ is the sum of a closed p-group and a divisible group.

Proof. Let $K$ be the closure of $H$ in $\bar{B}$. Since $\{G, K\} / G$ is divisible, $\{G, K\}$ is a pure subgroup of $\bar{B}$. If $S$ is a distinguished subsocle of $G$, then

$$
\{G, K\}[p] \supseteq\{G[p], K[p]\}=\{G[p], S\}=\bar{B}[p],
$$

and consequently $\{G, K\}=\bar{B}$. Then $G /(G \cap K) \cong \bar{B} / K$ is a closed $p$-group, and $(G \cap K) / H$ is divisible since it is pure in $K / H$. It remains only to observe that $G / H \cong(G \cap K) / H+G /(G \cap K)$.

Conversely, suppose that $G / H$ is the sum of a closed $p$-group and a divisible group. Since $(G \cap K) / H=(G / H)^{1}$ is divisible, $G \cap K$ is a pure subgroup of $G$. Also, $\{G, K\} / K=G /(G \cap K)$ is a closed p-group. But then $\{G, K\} / K$ is a direct summand of $\bar{B} / K$; and, since $\bar{B} / G$ is divisible, it follows that $\{G, K\}=\bar{B}$. From the purity of $G \cap K$, we have 


$$
\bar{B}[p]=\{G, K\}[p]=\{G[p], K[p]\}=\{G[p], S\} ;
$$

and $S$ is a distinguished subsocle of $G$.

Definition 3.6. A subsocle $S$ of $G$ is said to be semi-dense if $p^{n} G \cap S$ is dense in $\left(p^{n} G\right)[p]$ for some positive integer $n$. Just as in the case of dense subsocles, it is evident that any semidense subsocle is distinguished. For subsocles of $G$ which are not semidense, we have the following.

Corollary 3.7. Let $S$ be a subsocle of $G$ which is not semidense. Then $S$ is a distinguished subsocle of $G$ if and only if the closure of $S$ in $G$ supports a pure subgroup $H$ of $G$ such that $G / H$ is an unbounded closed p-group.

Proof. Let $S^{*}$ be the closure of $S$ in $G$. Then $S$ is distinguished if and only if $S^{*}$ is. Since $S$ is not semidense, neither is $S^{*}$. Now $S^{*}$ is distinguished if and only if it supports a pure subgroup $H$ of $G$ such that $G / H$ is the sum of a closed $p$-group and a divisible group. Since $S^{*}$ is closed in $G, H$ is likewise closed in $G$ and therefore $(G / H)^{1}=0$. Thus, $S^{*}$ is distinguished if and only if $G / H$ is a closed $p$-group. Finally, $G / H$ is unbounded since $S^{*}$ is not semidense.

We conclude this section with a lemma which is needed to establish the main theorem of the next section.

LEMMA 3.8. Let $K$ be a closed p-group with a countable basic subgroup and suppose that $S$ is a proper dense subsocle of $K$ having cardinality $c$. Then $S$ supports $2^{c}$ isomorphically distinct pure subgroups $G$ of $K$ such that no nondistinguished, closed subsocle of $G$ having cardinality $c$ supports a pure subgroup of $G$.

Proof. Let $\mathscr{H}_{0}$ be the collection of all direct summands $H$ of $K$ such that $|p H \cap S|=c$ and $\{H[p], S\} \neq K[p]$. Since $K$ has only $c$ distinct direct summands, $\left|\mathscr{H}_{0}\right| \leqq c$. Now let $\mathscr{H}_{1}$ be a collection of $c$ distinct pure subgroups of $K$ supported by $S$ and let $\mathscr{H}$ be the union of $\mathscr{H}_{0}$ and $\mathscr{H}_{1}$. Since $|\mathscr{H}|=c$, by Lemma 2.1, there are $2^{c}$ linearly independent subsets $L=\left[k_{H}\right]_{H \in \mathscr{H}}$ of $K$ such that for each $H \in \mathscr{H}:$ (a) $O\left(k_{H}\right)=p^{2}$, (b) $k_{H} \in\{H, K[p]\}$, (c) $p k_{H} \in S$, (d) $k_{H} \notin\{H, S\}$, and such that no two distinct such subsets $L$ generate with $G[p]$ the same subgroup of $G$. For each $L$, let $G$ be a pure subgroup of $K$ such that $L \subseteq G$ and such that $G[p]=S$. Now suppose that $T$ is a nondistinguished, closed subsocle of $G$ having cardinality $c$. Assume that $T$ supports a pure subgroup $A$ of $G$ and let $H$ be the closure of $A$ in $K$. Then $H$ is a member of $\mathscr{H}_{0} \subseteq \mathscr{H}$. Since $T$ is closed in $G, A$ is likewise closed in $G$ and therefore $A=G \cap H$. The purity of $A$ then implies that

$$
\{H, G\}[p]=\{H[p], G[p]\}=\{H[p], S\} .
$$

By (b), there is a $z \in K[p]$ and an $h \in H$ such that $k_{H}=h+z$. Then

$$
z \in\{H, G\}[p]=\{H[p], S\},
$$


which implies that $k_{H} \in\{H, S\}$, in contradiction to (d). We conclude that $T$ can support no pure subgroup of $G$. Since we have $2^{c}$ such pure subgroups $G$ of $K$ the proof of the lemma is complete.

4. Essentially indecomposable groups. Examples of unbounded reduced primary groups which are essentially indecomposable have been given previously in [1], [7], and [12]. We are now in position to prove rather general existence theorems for such groups.

THEOREM 4.1. Let $K$ be a closed p-group with a countable basic subgroup and suppose that $S$ is a proper dense subsocle of $K$ having cardinality $c$. Then $S$ supports $2^{c}$ isomorphically distinct essentially indecomposable pure subgroups of $K$.

Proof. By Lemma 3.8, there is a collection $\mathscr{E}$ of $2^{c}$ isomorphically distinct pure subgroups of $K$ supported by $S$ such that each member $G$ of $\mathscr{E}$ has the property that if $T$ is any nondistinguished, closed subsocle of $G$ having cardinality $c$, then $T$ does not support a pure subgroup of $G$. The proof of the theorem is completed by the observation that our Theorem 3.5 implies that any such $G$ is essentially indecomposable.

The following theorem can also be established by appropriately modifying Lemma 3.8 .

THEOREM 4.2. Let $G$ be a primary group without elements of infinite height having a countable basic subgroup and suppose that $S$ is a proper dense subsocle of $G$ having cardinality $c$. Then $S$ supports a pure subgroup $H$ of $G$ such that if $H=H_{1}+H_{2}$, then one of $H_{1}$ and $H_{2}$ is a direct summand of $G$ having cardinality less than $c$.

COROLlary 4.3. Let $K$ be a closed p-group with a countable basic subgroup and suppose that $G$ is a dense pure subgroup of $K$ such that $|K / G|=\aleph_{0}$. If $S$ is a proper dense subsocle of $G$ having cardinality $c$, then $S$ supports a pure subgroup of $G$ which is essentially indecomposable.

5. Pure-complete groups. A primary group will be said to be pure-complete if each of its subsocles supports a pure subgroup. Direct sums of cyclic groups and quasi-closed groups are examples of pure-complete groups. It is clear that no reduced primary group with elements of infinite height can be pure-complete. An example of a primary group without elements of infinite height which is not pure-complete was given in [11] and obviously our Lemma 3.8 implies the existence of such groups. Following Kolettis [10], we call a primary group semicomplete if it is the direct sum of a closed $p$-group and a direct sum of cyclic groups. It was shown in [6] that any semicomplete primary group is pure-complete In this section, we generalize the just cited result in various ways.

LEMMA 5.1. Suppose that $G$ and $H$ are primary groups with $H$ bounded and 
let $S$ be a subsocle of $G+H$. If $A$ is a pure subgroup of $G$ supported by $S \cap G$, then $S$ supports a pure subgroup of $G+H$ which contains $A$.

Proof. $\{S, A\} \mid A$ is a discrete subsocle of $(G+H) / A$ and hence supports a pure subgroup $A^{\prime} \mid A$ of $(G+H) / A$. Then $A^{\prime}[p]=S$ and $A^{\prime}$ is pure in $G+H$ since $A$ and $A^{\prime} \mid A$ are pure subgroups of $G+H$ and $(G+H) / A$ respectively.

A consequence of this lemma is

THeOREM 5.2. If $G$ is pure-complete and if $H$ is a direct sum of cyclic groups, then $G+H$ is pure-complete.

Proof. Since $H$ is a direct sum of cyclic groups, $G+H$ is the union of a monotone sequence of pure subgroups $G+H_{n}$ with $H_{n}$ bounded. Let $S$ be a subsocle of $G+H$ and set $S_{n}=S \cap\left(G+H_{n}\right)$. Using Lemma 5.1 and the fact that $G$ is pure-complete, we can construct a monotone sequence of pure subgroups $A_{n} \subseteq G+H_{n}$ such that $A_{n}[p]=S_{n}$. Then $A=\bigcup_{n=1}^{\infty} A_{n}$ is a pure subgroup of $G+H$ supported by $S$.

THEOREM 5.3. If $G$ is pure-complete and if $H$ is a closed p-group with finite Ulm invariants, then $G+H$ is pure-complete.

Proof. We may assume that $G$ is without elements of infinite height. Now let $S$ be a subsocle of $G+H$ and let $S^{\prime}$ be the projection of $S$ into $G$. Since $G$ is purecomplete, there is a pure subgroup $A$ of $G$ supported by $S^{\prime}$. Then $S$ is contained in the pure subgroup $A+H$, and it clearly suffices to show that $S$ is a distinguished subsocle of $A+H$. Let $\bar{B}$ be the torsion completion of $A+H$ and note that $\bar{B}=\bar{A}+H$, where $\bar{A}$ is the closure of $A$ in $\bar{B}$. Since $H$ is a closed $p$-group with finite Ulm invariants, its socle is compact. But then $\{S, H[p]\}$ contains $\bar{S}^{\prime}=\bar{A}[p]$. Therefore $\bar{B}[p]=\{S, H[p]\}$ and $S$ is distinguished.

COROLlaRY 5.4. If $G$ is pure-complete and if $H$ is a semicomplete primary group with finite Ulm invariants, then $G+H$ is pure-complete.

In spite of the foregoing results and the easily established fact that a direct summand of a pure-complete group is pure-complete, the direct sum of two purecomplete groups need not be pure-complete.

EXAMPLE 5.5. Suppose that $K$ is an unbounded closed $p$-group with a countable basic subgroup. Then $K$ contains a proper, dense pure subgroup $G$ such that $G$ is a quasi-closed primary group and $|G[p]|=c$ (see [7]). By Theorem 2.3, $K$ contains a pure subgroup $H$ such that $H[p]=G[p]$ and $H \nsubseteq G$. By Corollary 3.3, $H$ is also quasi-closed and therefore both $G$ and $H$ are pure-complete. However, the subsocle $S$ of $G+H$ consisting of alle elements of the form $(x, x)$ with $x \in G[p]=H[p]$ does not support a pure subgroup of $G+H$. Indeed, if $A$ is a pure subgroup of $G+H$ supported by $S$, then it is easily seen that $A$ is a sub- 
direct sum of $G$ and $H$ with zero kernels, contrary to the fact that $G$ and $H$ are not isomorphic.

We can prove one further result which, in a certain sense, generalizes the fact that semicomplete groups are pure-complete.

THEOREM 5.6. If $G_{n}$ is quasi-closed and a pure subgroup of $G_{n+1}$ for each positive integer $n$, then $G=\bigcup_{n=1}^{\infty} G_{n}$ is pure-complete.

Proof. Let $S$ be a subsocle of $G$ and set $S_{n}=S \cap G_{n}$. Now if each $S_{n}$ is discrete, then $S=\bigcup_{n=1}^{\infty} S_{n}$ supports a direct sum of cyclic groups which is pure in $G$. Otherwise, Corollary 3.4 can be applied to complete the proof in a manner similar to the proof of Theorem 5.2.

In spite of the fact that most primary groups with rather simple structure seem to be pure-complete, it appears that pure-complete groups are exceptional rather than the rule. In particular, we do not know the answer, even in the case of countable basic subgroups, to the following question.

Problem 5.7. Does every dense subsocle of a closed p-group support a pure subgroup which is pure-complete?

6. Infinite primary groups without proper isomorphic subgroups. We shall say that a group is without proper isomorphic subgroups if it is isomorphic to no proper subgroup of itself. In order that an infinite reduced primary group be without proper isomorphic subgroups it is necessary that it be unbounded, uncountable, and have finite Ulm invariants. Crawley [2] and Pierce [12] have indeed constructed infinite reduced primary groups without proper isomorphic subgroups, and the purpose of this section is to give a more general and simpler construction of such groups.

LEMMA 6.1. If $\phi$ is an endomorphism of a closed p-group $K$ such that $|\phi(K[p])|<c$, then $\left(p^{n} K\right)[p] \subseteq \operatorname{ker} \phi$ for some integer $n$.

Proof. If $\left(p^{n} K\right)[p] \notin \operatorname{ker} \phi$ for all $n$, then we can choose a sequence $x_{1}, x_{2}, \cdots$ of elements in $K[p]$ such that $x_{i} \notin \operatorname{ker} \phi$ and $h\left(x_{i+1}\right)>h\left(\phi\left(x_{i}\right)\right)$ for each $i$. Let $y_{i}=\phi\left(x_{i}\right)$ and observe that the $y_{i}$ form a linearly independent subset of $K[p]$. Set $S=\sum_{i=1}^{\infty}\left\{y_{i}\right\}$ and let $S$ denote the closure of $S$ in $K$. Then $|S|=c$ and it is easily seen that $S \subseteq \phi(K[p])$.

Corollary 6.2. If $\phi$ is an endomorphism of a closed p-group $K$ and if $S$ is a subsocle of $K$ such that $|K[p] / S|<c$ and $|\phi(S)|<c$, then $(p K)[p] \subseteq \operatorname{ker} \phi$ for some integer $n$.

THEOREM 6.3. If $K$ is a closed p-group with finite Ulm invariants and if $S$ is a proper dense subsocle of $K$ such that $|K[p] / S|<c$, then $S$ supports a pure subgroup of $K$ which is without proper isomorphic subgroups.

Proof. Consider all endomorphisms $\phi$ of $K$ such that (1) $\phi \mid S$ is one-to-one 
(2) $\phi(S) \subseteq S$ and (3) for each $n, \phi \mid S \cap p^{n} K$ is not multiplication by a fixed constant. Then index this collection of all such endomorphisms $\phi_{\alpha}$ by ordinals $\alpha<\mu$, where $\mu$ does not exceed the first ordinal of cardinality $c$. Let $S=S_{0}+(S \cap p K)$ and choose $z$ in $K[p]$ but not in $S$. We wish to find, for each $\alpha<\mu$, an $x_{\alpha}$ and a $y_{\alpha}$ in $K$ such that (i) $O\left(x_{\alpha}\right)=O\left(y_{\alpha}\right)=p^{2}$, (ii) $p x_{\alpha}, p y_{\alpha} \in S$, (iii) $\phi_{\alpha}\left(x_{\alpha}\right)=y_{\alpha}+z$ and (iv) the collection of all the $x_{\alpha}, y_{\alpha}$ forms a linearly independent subset of $K$.

Suppose that $\beta<\mu$ and assume that the $x_{\alpha}, y_{\alpha}$ with the desired properties have been chosen for all $\alpha<\beta$. Let $H=S_{0}+p\left(\Sigma_{\alpha<\beta}\left(\left\{x_{\alpha}\right\}+\left\{y_{\alpha}\right\}\right)\right)$ and choose $V \subseteq S \cap p K$ such that $S=H+V$. Observe that $|H|<c$ and $|K[p] / V|<c$. We wish to find a nonzero $x \in V$ such that $\left\{H, x, \phi_{\beta}(x)\right\}=H+\{x\}+\left\{\phi_{\beta}(x)\right\}$. Assume that no such $x$ exists. Then for each nonzero $x \in V$ there exists a unique $h \in H$ and a unique nonnegative integer $t<p$ such that $\phi_{\beta}(x)=h+t x$. It is easily seen that $t$ is independent of $x$ and consequently the endomorphism $\psi=t-\phi_{\beta}$ is such that $\psi(V) \subseteq H$. From Corollary 6.2 , we conclude that $\left(p^{n} K\right)[p] \subseteq \operatorname{ker} \psi$ for some $n$, which however contradicts (3). Therefore there must exist a nonzero $x \in V$ such that $\left\{H, x, \phi_{\beta}(x)\right\}=H+\{x\}+\left\{\phi_{\beta}(x)\right\}$. We need only choose $x_{\beta} \in K$ such that $p x_{\beta}=x$ and set $y_{\beta}=\phi_{\beta}\left(x_{\beta}\right)-z$.

Now let $G$ be a subgroup of $K$ which contains $\Sigma_{\alpha<\mu}\left(\left\{x_{\alpha}\right\}+\left\{y_{\alpha}\right\}\right)$ and is maximal in $K$ with respect to $G[p]=S$. $G$ is then a pure subgroup of $K$ with $K / G$ divisible. Observe that $\phi_{\alpha}(G) \nsubseteq G$ for each $\alpha<\mu$ since $\phi_{\alpha}\left(x_{\alpha}\right) \notin G$. Suppose $\phi$ is a one-to-one endomorphism of $G$. Since $\phi$ extends uniquely to an endomorphism of $K$, we may assume that $\phi$ is itself an endomorphism of $K$. Since $\phi$ satisfies (1) and (2), but $\phi \neq \phi_{\alpha}$ for each $\alpha<\mu$, there are integers $n$ and $t$ such that $0<t<p$ and $\phi(x)=t x$ for all $x \in S \cap p^{n} K=\left(p^{n} G\right)[p]$. Let $G=A+M$, where $M[p]=\left(p^{n} G\right)[p]$ and $A$ is necessarily finite. Since $\phi \mid M[p]$ is multiplication by an integer prime to $p$, $\phi(M)$ is a pure subgroup of $G$ having the same socle as $M$. Hence $G=A+\phi(M)$ and $\phi(G)=(A \cap \phi(G))+\phi(M)$. But

$$
A \cong G / M \cong \phi(G) / \phi(M) \cong A \cap \phi(G)
$$

and, since $A$ is finite, we conclude that $A=A \cap \phi(G)$ and $G=\phi(G)$.

7. Reduced primary groups larger than their basic subgroups. Khabbaz [9] calls a primary group starred if it satisfies any of several equivalent conditions, one of which being that it has the same cardinality as its basic subgroups. Assuming the generalized continuum hypothesis, we can generalize certain of our resultsTheorem 2.3, in particular-for reduced primary groups which are not starred. Indeed, using the techniques of $\S 2$, we can prove

THEOREM 7.1. If $G$ is a reduced primary group containing a basic subgroup $B$ such that $2^{|B|}<2^{|G|}$, and if $S$ is a proper dense subsocle of $G$ having the same cardinality as $G$, then $S$ supports $2^{|G|}$ isomorphically distinct pure subgroups of $G$. 
Similarly, an appropriate generalization of Theorem 4.2 can be established. However, in this more general context, specialization to closed $p$-groups no longer leads to the existence of essentially indecomposable groups.

\section{REFERENCES}

1. R. A. Beaumont and R. S. Pierce, Some invariants of p-groups, Michigan Math. J. 11 (1964), 137-149.

2. Peter Crawley, An infinite primary abelian group without proper isomorphic subgroups, Bull. Amer. Math. Soc. 68 (1962), 462-467.

3. L. Fuchs, Abelian groups, Publ. House Hungarian Academy of Sciences, Budapest, 1958.

4. Paul Hill, Certain pure subgroups of primary groups, Topics in Abelian Groups, Scott, Foresman and Co., Chicago, IIl., 1963.

5. —_ Pure subgroups having prescribed socles, Bull. Amer. Math. Soc. 71 (1965), 608-609.

6. Paul Hill and Charles Megibben, Minimal pure subgroups in primary groups, Bull. Soc. Math. France 92 (1964), 251-257.

7. —, Quasi-closed primary groups, Acta Math. Acad. Sci. Hungar. 16 (1965), 271-274.

8. J. Irwin, C. Peercy and E. Walker, Splitting properties of high subgroups, Bull. Soc. Math. France 90 (1962), 185-192.

9. S. A. Khabbaz, Abelian torsion groups having a minimal system of generators, Trans. Amer. Math. Soc. 98 (1961), 527-538.

10. G. Kolettis, Semi-complete primary abelian groups, Proc. Amer. Math. Soc. 11 (1960), 200-205. 453-454.

11. C. Megibben, A note on a paper of Bernard Charles, Bull. Soc. Math. France 91 (1963),

12. R. S. Pierce, Homomorphisms of primary abelian groups, Topics in Abelian Groups, Scott, Foresman and Co., Chicago, Ill., 1963.

EMORY UNIVERSITY, Atlanta, Georgia

UNIVERSITY OF WASHINGTON, SeATtLe, Washington 